\title{
EVERY WEAK PROPER HOMOTOPY EQUIVALENCE IS WEAKLY PROPERLY HOMOTOPIC TO A PROPER HOMOTOPY EQUIVALENCE
}

BY

\author{
DAVID A. EDWARDS AND HAROLD M. HASTINGS( ${ }^{1}$ )
}

\begin{abstract}
We prove that every weak proper homotopy equivalence of $\sigma$-compact, locally compact Hausdorff spaces is weakly properly homotopic to a proper homotopy equivalence.
\end{abstract}

In $[6$, pp. $489-491]$, L. Siebenmann obtained a convenient criterion $\left(\pi_{*}\right)_{\infty}$ for a proper map of finite dimensional polyhedra (polyhedron $=$ locally finite simplicial complex) to be a proper homotopy equivalence. Later, E. M. Brown [1, p. 23] , and F. T. Farrell, L. R. Taylor, and J. B. Wagoner [5, Theorem 3.5] claimed to be able to remove the finite dimensional assumption. In [4] we give an example which shows that the finite dimensional assumption is necessary. On the positive side, we prove in $\S 2$ the following useful (see [2], [8]) theorem.

(1.1) TheOREM. Let $f: X \rightarrow Y$ be a proper map of o-compact, locally compact Hausdorff spaces. If $f$ is a weak proper homotopy equivalence, then $f$ is weakly properly homotopic to a proper homotopy equivalence.

(1.2) Remarks. Chapman and Siebenmann, in developing an obstruction theory for putting boundaries on noncompact $Q$-manifolds ( $Q$ denotes the Hilbert cube) [8], asked two questions.

(1) Is every weak proper homotopy equivalence a proper homotopy equivalence?

(2) Is every weak proper homotopy equivalence weakly properly homotopic to a proper homotopy equivalence?

They obtained the following independent results in [8]. For finite dimensional polyhedra, (1) holds by Siebenmann's $\left(\pi_{*}\right)_{\infty}$ criterion. For polyhedra with tame

Received by the editors June 19, 1975.

AMS (MOS) subject classifications (1970). Primary 55D10; Secondary 55B99.

Key words and phrases. Pro-homotopy theory, proper homotopy theory, Whitehead Theorems, $Q$-manifolds.

( $\left.{ }^{1}\right)$ This paper was written while the second-named author held a visiting position at the State University of New York at Binghamton, whose hospitality he gratefully acknow. ledges. 
ends, (2) holds. This uses [7], [8], [9], [10], and [11]. (Note that any $Q$-manifold can be triangulated as $X \times Q$ for some polyhedron $X$, and conversely [7], [11].)

In [3] we develop machinery which allows us to prove that a proper map which is a homotopy equivalence and a homotopy equivalence at $\infty$ (appropriately defined) is a proper homotopy equivalence.

ACKNOWLEDGEMENTS. We wish to thank Ross Geoghegan for helpful discussions.

2. Proof of Theorem. We begin by extending Chapman's definition of proper map to a definition of filtered map of filtered spaces. A filtered space $X$ consists of a space $X$, together with a sequence of closed subspaces

$$
X=X_{0} \supset X_{1} \supset X_{2} \supset \cdots,
$$

with each $X_{n-1} \subset$ int $X_{n}$. A map $f: X \rightarrow Y$ of filtered spaces is called a filtered map if for each number $n \geqslant 0$ there exists a number $m \geqslant 0$ with $f\left(X_{m}\right) \subset Y_{n}$. A filtered space $X$ induces a natural filtration on its cylinder $X \times I$; this yields a natural notion of filtered homotopy. There results a filtered category and its filtered homotopy category.

The connection with the proper homotopy theory of $\sigma$-compact, locally compact (Hausdorff) spaces (for example, countable locally finite simplicial complexes) is made as follows. Suppose $X=\bigcup_{n=0}^{\infty} K_{n}$ where $K_{0}=\varnothing$, each $K_{n}$ is compact, and each $K_{n-1} \subset$ int $K_{n}$. We associate to $X$ the filtered space

$$
\begin{gathered}
\epsilon(X)=\left\{X=X_{0} \supset X_{1} \supset X_{2} \supset \cdots \cdot,\right. \text { where } \\
X_{n}\left(=(\epsilon(X))_{n}\right)=\operatorname{cl}\left(X \backslash K_{n}\right) .
\end{gathered}
$$

$\epsilon(X)$ is called the end of $X$. Ends are unique up to canonical filtered isomorphism. Clearly, a map $f: X \rightarrow Y$ of $\sigma$-compact, locally compact spaces is proper (following Chapman) if and only if $f$ induces a filtered map $\epsilon(f): \epsilon(X) \rightarrow \epsilon(Y)$.

We associate to an end the following mapping telescope. The telescope of a diagram

$$
\bar{X}=\left\{X_{0} \stackrel{f_{1}}{\longleftarrow} X_{1} \stackrel{f_{2}}{\longleftarrow} X_{2} \stackrel{f_{3}}{\longleftarrow} \ldots\right\}
$$

is the space

$$
\operatorname{Tel}(\bar{X})=X_{0} \times 0 \cup_{f_{1}} X_{1} \times[0,1] \cup_{f_{2}} X_{2} \times[1,2] \cup_{f_{3}} \cdots,
$$

which is filtered by setting

$$
\operatorname{Tel}(\bar{X})_{n}=X_{n} \times n \cup_{f_{n+1}} X_{n+1} \times[n, n+1] \cup_{f_{n+2}} \cdots,
$$

for $n \geqslant 0$. 


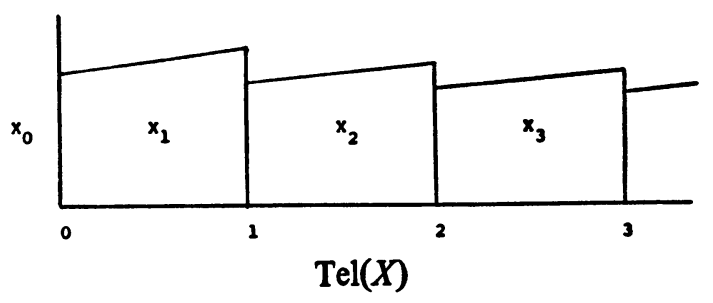

Let $X$ be a $\sigma$-compact, locally compact space, with $X=\bigcup_{n=0}^{\infty} K_{n}, K_{0}=$ $\varnothing$, each $K_{n}$ compact, and $K_{n-1} \subset$ int $K_{n}$. There is a natural projection $p: \operatorname{Tel}(\epsilon(X)) \rightarrow X\left(\epsilon(X)\right.$ is bonded by the inclusions $\left.f_{i}\right)$; clearly $p$ is a filtered map. A proper section for $\epsilon(X)$ is a filtered map s: $X \rightarrow \operatorname{Tel}(\epsilon(X))$ with $p s=\mathrm{id}_{x}$.

(2.1) Construction of proper sections. The Tietze extension theorem yields maps $h_{n}: \operatorname{cl}\left(K_{n} \backslash K_{n-1}\right) \rightarrow[0,1]$ with $h_{n}\left(\right.$ bd $\left.K_{n-1}\right)=n-2$ and $h_{n}\left(\right.$ bd $\left.K_{n}\right)=$ $n-1$ for $n \geqslant 2$. We may glue these maps together to obtain a proper map $h: X \rightarrow R^{+}$(notation: $R^{+}$denotes the set of nonnegative real numbers) such that $h\left(K_{n} \backslash K_{n-1}\right) \subset[n-2, n-1]$ for $n \geqslant 1$. Because $\left(X_{n-1} \backslash X_{n}\right) \subset \mathrm{cl}\left(K_{n} \backslash K_{n-1}\right)$, there results a map $s: X \rightarrow \operatorname{Tel}(\epsilon(X))$, given by the formula

$$
s(x)=(x, h(x)) \text {. }
$$

Clearly, $s$ is a proper section for $\epsilon(X)$. In fact, each proper section $s^{\prime}$ for $\epsilon(X)$ comes from a suitable proper map $h^{\prime}: X \rightarrow R^{+}$and formula (2.2).

Finally, we introduce a relation of vertical homotopy into the set of maps $\{X \rightarrow \operatorname{Tel}(\epsilon(Y))\}$. Let $p: \operatorname{Tel}(\epsilon(y)) \rightarrow Y$ denote the projection. Maps $f_{0}, f_{1}$ : $X \rightarrow \operatorname{Tel}(\epsilon(Y))$ with $p f_{0}=p f_{1}=f: X \rightarrow Y$ are called vertically homotopic if there is a homotopy $H=\left\{H_{t}\right\}: X \times I \rightarrow \operatorname{Tel}(\epsilon(Y))$ with $H_{0}=f_{0}, H_{1}=f_{1}$, and $p H_{t}=f$ for all $t$. We call $H$ a vertical homotopy. If $f_{0}, f_{1}$, and $H$ are also filtered maps, $f_{0}$ and $f_{1}$ are called filtered-vertically-homotopic. Clearly, any two proper sections for $\epsilon(X)$ are filtered-vertically-homotopic.

We may now begin the proof of Theorem 1.1. Let $f: X \rightarrow Y$ be a weak proper homotopy equivalence of $\sigma$-compact, locally compact (Hausdorff) spaces. Choose a proper map $g: Y \rightarrow X$ which is a weak-proper-homotopy inverse to $f$. Then choose ends

$$
\epsilon(X)=\left\{X=X_{0} \supset X_{1} \supset X_{2} \supset \cdots\right\}, \epsilon(Y)=\left\{Y=Y_{0} \supset Y_{1} \supset Y_{2} \supset \cdots \bullet,\right.
$$

such that

(i) $X_{n} \subset \operatorname{int}\left(X_{n-1}\right)$ and $Y_{n} \subset \operatorname{int}\left(Y_{n-1}\right)$ for $n \geqslant 1$,

(ii) $f\left(X_{n}\right) \subset Y_{n}$ for $n \geqslant 0$,

(iii) $g\left(Y_{n}\right) \subset X_{n-1}$ for $n \geqslant 1$,

(iv) there exist homotopies $H_{n}: X \times I \rightarrow X$ with $H_{n} l_{0}=$ id and $H_{n} l_{1}=g f$ for $n \geqslant 0$, and further, $H_{n}\left(X_{n} \times I\right) \subset X_{n-1}$ for $n \geqslant 1$, and 
(v) There exists homotopies $K_{n}: Y \times I \rightarrow Y$ with $\left.K_{n}\right|_{0}=$ id and $\left.K_{n}\right|_{1}=f g$ for $n \geqslant 0$, and further, $K_{n}\left(Y_{n} \times I\right) \subset Y_{n-1}$ for $n \geqslant 1$.

(Such ends are easily obtained by successively passing to cofinal subsystems of any ends of $X$ and $Y$.)

We shall use the above data to construct a suitable proper homotopy equivalence $f^{\prime}: X \rightarrow Y$. Write $f_{n}$ for $\left.f\right|_{X_{n}}$ and $g_{n}$ for $\left.g\right|_{Y_{n}}$. Let $\bar{Z}$ be the inverse system

$$
Y_{0} \stackrel{f_{0}}{\longleftarrow} X_{0} \stackrel{g_{1}}{\longleftarrow} Y_{1} \stackrel{f_{1}}{\longleftarrow} X_{1} \stackrel{g_{2}}{\longleftarrow} \ldots .
$$

Form the homotopy-commutative diagram
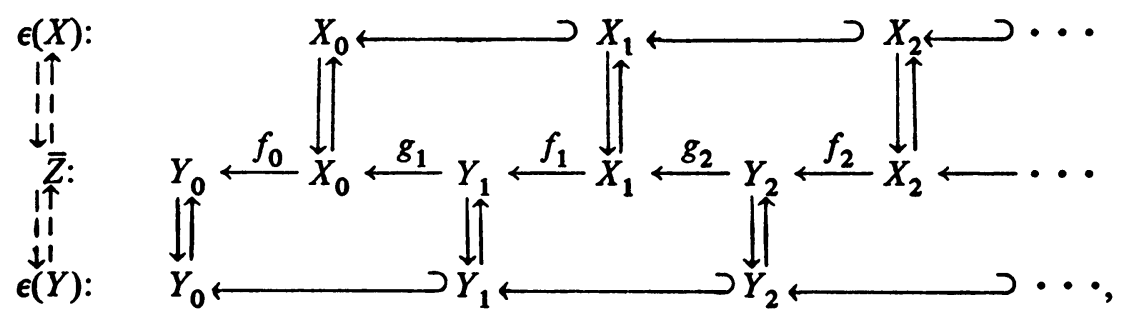

in which the vertical arrows denote the appropriate identity maps, and the required homotopies are given by conditions (iv) and (v) above.

Diagram (2.3), together with the homotopies (iv) and (v), yields filtered (but not proper) maps of mapping telescopes

$$
\operatorname{Tel}(\epsilon(X)) \underset{F^{\prime}}{\stackrel{F}{\rightleftarrows}} \operatorname{Tel}(\bar{Z}) \underset{G}{\stackrel{G^{\prime}}{\rightleftarrows}} \operatorname{Tel}(\epsilon(Y)) .
$$

In order to give explicit formulas for these maps, we regard

$$
\operatorname{Tel}\left(w_{0} \stackrel{h_{0}}{\longleftarrow} w_{1} \stackrel{h_{1}}{\longleftarrow} w_{2} \stackrel{h_{2}}{\longleftarrow} \ldots\right)
$$

as the union of the "levels" $W_{n-1} \times 0 \cup_{h_{n-1}} W_{n} \times[0,1]$. Then $F$ maps $X_{n-1}$ $\times 0 \cup X_{n} \times[0,3 / 4]$ to $X_{n-1} \times 0 \cup_{g_{n}} Y_{n} \times[0,1]$ by the formula

$$
F(x, t)= \begin{cases}\left(H_{n}(x, 2 t), 0\right) \in X_{n-1} \times 0 & \text { for } 0 \leqslant t \leqslant 1 / 2, \\ \left(g_{n} f_{n}(x), 0\right) \in X_{n} \times 0 & \text { for } t=1 / 2, \\ \left(f_{n}(x), 4 t-2\right) \in Y_{n} \times[0,1] & \text { for } 1 / 2 \leqslant t \leqslant 3 / 4,\end{cases}
$$

and $F$ maps $X_{n} \times[3 / 4,1]$ to $Y_{n} \times 0 \cup_{f_{n}} X_{n} \times[0,1]$ by the formula

$$
F(x, t)= \begin{cases}\left(f_{n}(x), 0\right) \in Y_{n} \times 0 & \text { for } t=3 / 4 \\ (x, 4 t-3) \in X_{n} \times[0,1] & \text { for } 3 / 4 \leqslant t \leqslant 1\end{cases}
$$

See Figure I. The map $G$ in diagram (2.4) has an analogous description. 

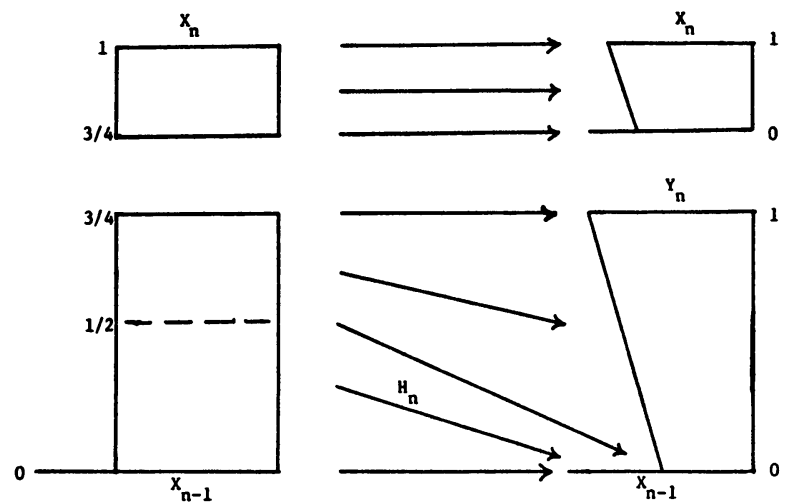

Figure I

The map $F^{\prime}: \operatorname{Tel}(\bar{Z}) \rightarrow \operatorname{Tel}(\epsilon(X))$ maps $X_{n-1} \times 0 U_{g_{n}} Y_{n} \times[0,1]$ into $X_{n-1} \times 0 \cup X_{n} \times[0,1]$ according to the formula

$$
\left\{\begin{array}{l}
F^{\prime}(x, 0)=(x, 0), \\
F^{\prime}(y, t)=\left(g_{n}(y), 0\right),
\end{array}\right.
$$

and maps $Y_{n} \times 0 \cup_{f_{n}} X_{n} \times[0,1]$ into $X_{n-1} \times 0 \cup X_{n} \times[0,1]$ according to the formula

$$
\left\{\begin{aligned}
F^{\prime}(y, 0) & =\left(g_{n}(y), 0\right), \\
F^{\prime}(x, t) & = \begin{cases}\left(g_{n} f_{n}(x), 0\right) & \text { for } t=0, \\
\left(H_{n}(x, 1-2 t), 0\right) & \text { for } 0 \leqslant t \leqslant 1 / 2, \\
(x, 2 t-1) & \text { for } 1 / 2 \leqslant t \leqslant 1\end{cases}
\end{aligned}\right.
$$

See Figure II. The function $(, t) \rightarrow\left(H_{n}(, 1-2 t), 0\right)$ used in the definition of $F^{\prime}$ is indicated by " $-H$ " in Figure II to indicate that $1-2 t$ decreases as $t$ increases. The map $G^{\prime}$ in diagram (2.4) has an analogous description.

Our construction also yields filtered homotopies

$$
\begin{aligned}
& F^{\prime} F \sim \mathrm{id}_{\operatorname{Tel}(\epsilon(X))}, F F^{\prime} \sim \mathrm{id}_{\operatorname{Tel}(Z)}, \quad \text { and } \\
& G^{\prime} G \sim \mathrm{id}_{\operatorname{Tel}(\epsilon(Y))}, G G^{\prime} \sim \mathrm{id}_{\operatorname{Tel}(\bar{Z})} .
\end{aligned}
$$

These homotopies arise from first deforming maps $H: X_{n} \times I \rightarrow X_{n-1}$ of the form $H_{n}+$ " $-H_{n}$ ":

$$
H(x, t)= \begin{cases}H_{n}(x, 2 t) & \text { for } 0 \leqslant t \leqslant 1 / 2, \\ H_{n}(x, 2-2 t) & \text { for } 1 / 2 \leqslant t \leqslant 1\end{cases}
$$

to the constant homotopy while keeping the top and bottom of the cylinder fixed, and then moving at most two levels within the telescopes. The first two homotopies are shown in Figures IIIa and IIIb. The formulas are complicated and not enlightening, and therefore omitted. 


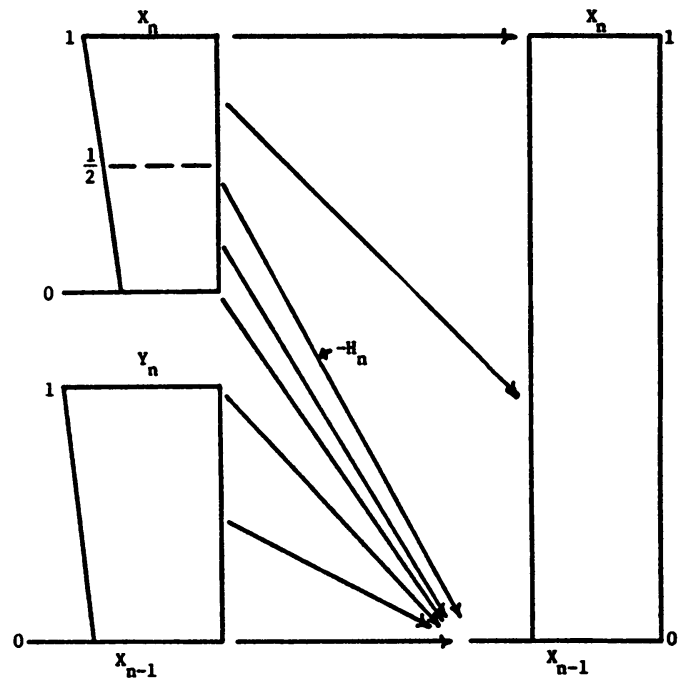

FIGURE II
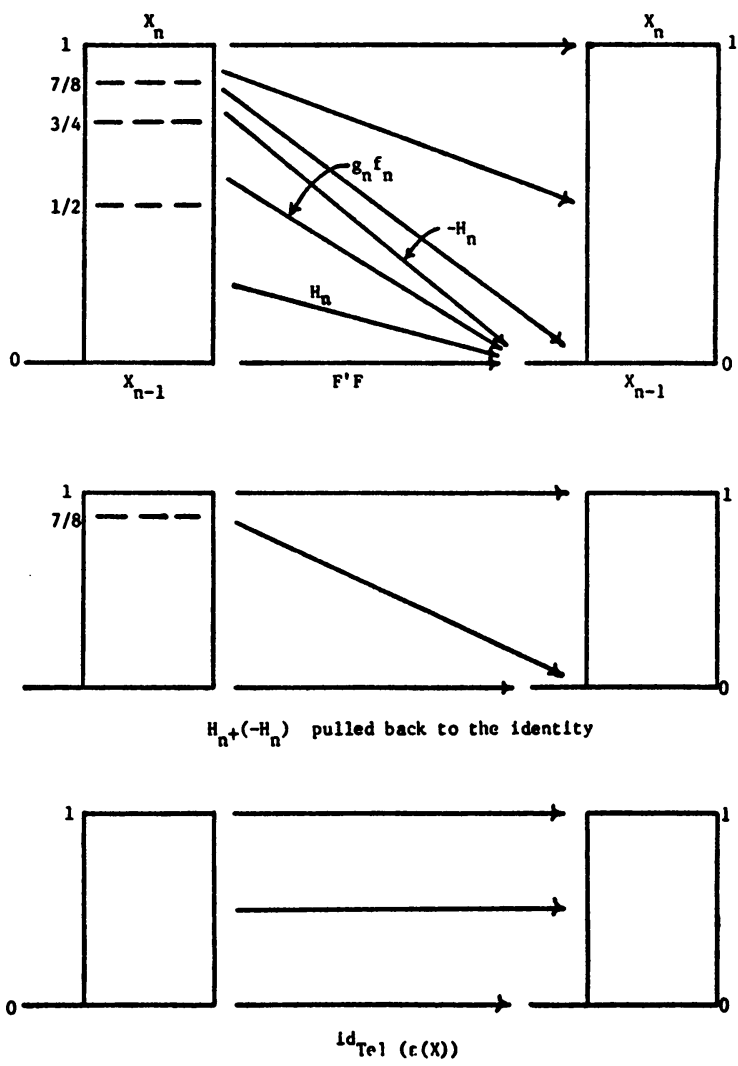

Deforming $F^{1} F$ to id Tel(e(X))

Figure IIIa 

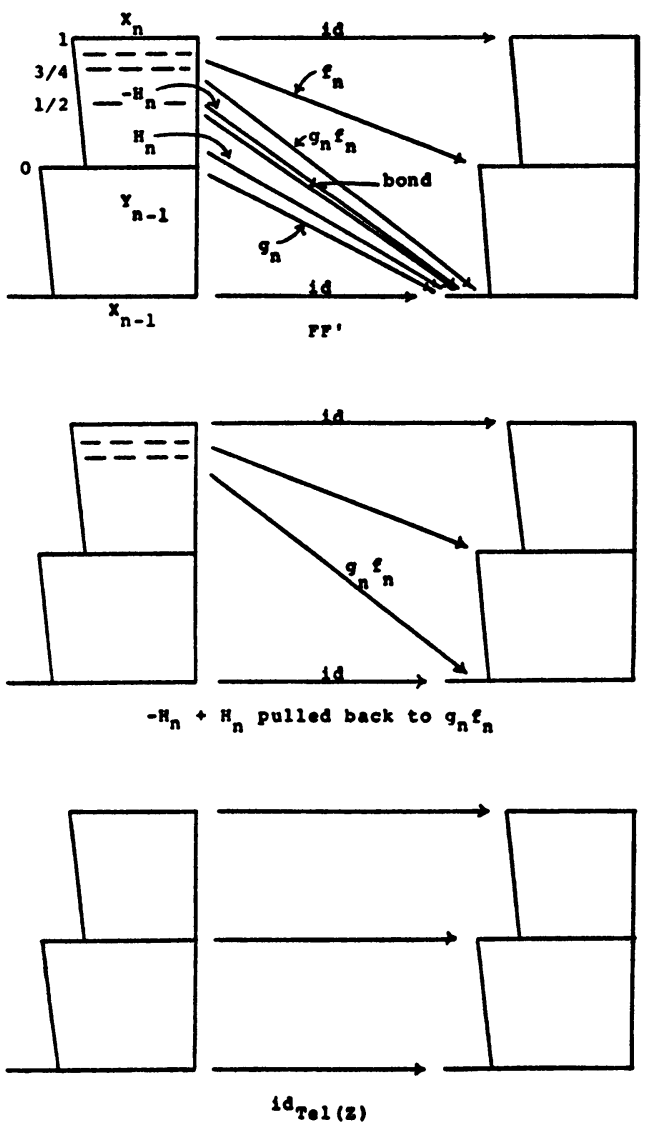

Deforming $F F^{\prime}$ to id $\mathrm{Tel(z)}$

FIGURE IIIb

Now write $p: \operatorname{Tel}(\epsilon(X)) \rightarrow X$ and $p^{\prime}: \operatorname{Tel}(\epsilon(Y)) \rightarrow Y$ for the projections. Choose proper maps $h: X \rightarrow R^{+}$and $h^{\prime}: Y \rightarrow R^{+}$with the property that $h\left(X_{n} \backslash X_{n-1}\right) \subset[n-2, n-1]$ and $h^{\prime}\left(Y_{n} \backslash Y_{n-1}\right) \subset[n-2, n-1]$ as in (2.1). (This is possible by property (i) of $\epsilon(X)$ and $\epsilon(Y)$.) As in formula (2.2), there result proper sections $s$ for $\epsilon(X)$ and $s^{\prime}$ for $\epsilon(Y)$, respectively. Define maps $f^{\prime}: X \rightarrow Y$ and $g^{\prime}: Y \rightarrow X$ to be the composites

$$
\left\{\begin{array}{l}
X \stackrel{s}{\longrightarrow} \operatorname{Tel}(\epsilon(X)) \stackrel{F}{\longrightarrow} \operatorname{Tel}(\bar{Z}) \stackrel{G^{\prime}}{\longrightarrow} \operatorname{Tel}(\epsilon(Y)) \stackrel{p^{\prime}}{\longrightarrow} Y, \\
Y \stackrel{s^{\prime}}{\longrightarrow} \operatorname{Tel}(\epsilon(Y)) \stackrel{G}{\longrightarrow} \operatorname{Tel}(\bar{Z}) \stackrel{F^{\prime}}{\longrightarrow} \operatorname{Tel}(\epsilon(X)) \stackrel{p}{\longrightarrow} X,
\end{array}\right.
$$

respectively. Because $f^{\prime}$ and $g^{\prime}$ are composites of filtered maps, they are filtered maps, and thus proper maps.

To complete the proof, we shall verify the following claims.

Claim 1. The maps $f^{\prime}$ and $g^{\prime}$ are proper homotopy inverses, hence the map $f^{\prime}: X \rightarrow Y$ is a proper homotopy equivalence. 
Claim 2. The maps $f^{\prime}, f: X \rightarrow Y$ are weakly-properly-homotopic.

Verification of Claim 1. Consider the commutative solid-arrow diagram

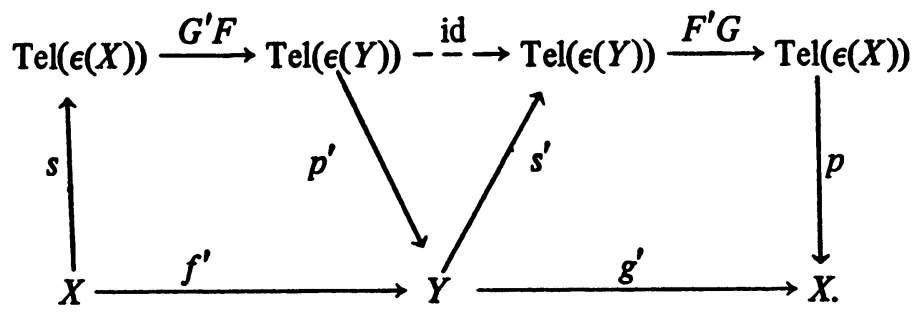

There exists a vertical homotopy $H$ between the maps

$$
H_{0} \equiv s^{\prime} p^{\prime} \circ G^{\prime} F \circ s, H_{1} \equiv G^{\prime} F \circ s: X \rightrightarrows \mathrm{Tel}(\epsilon(Y))
$$

given by the formula

$$
H(x, \tau)=\left(f^{\prime}(x),(1-\tau) \cdot \pi\left(H_{0}(x)\right)+\tau \cdot \pi\left(H_{1}(x)\right)\right)
$$

where $\pi$ denotes the projection $\operatorname{Tel}(\epsilon(Y)) \rightarrow R^{+}$. Because

$$
\pi H_{0}(x)=h^{\prime} f^{\prime}(x) \text { and }\left|\pi H_{1}(x)-h(x)\right| \leqslant 2
$$

by construction, $\pi H_{1}$ is a proper map and $H$ is a filtered vertical homotopy.

Hence the maps

$$
g^{\prime} f^{\prime}, p \circ F^{\prime} G \circ G^{\prime} F \circ s: X \rightrightarrows X
$$

are properly homotopic. But the composite $F^{\prime} \circ G G^{\prime} \circ F$ is filtered-homotopic to $\mathrm{id}_{\mathrm{Tel}(\epsilon(X))}$ by composites of the filtered homotopies of formula (2.5). Hence the map $g^{\prime} f^{\prime}$ is properly homotopic to id $X$. Similarly, $f^{\prime} g^{\prime}$ is properly homotopic to id ${ }_{Y}$, and Claim 1 follows.

Verification of Claim 2. The construction of the telescopes $\operatorname{Tel}(\epsilon(X))$, $\operatorname{Tel}(\epsilon(Y))$ and $\operatorname{Tel}(\bar{Z})$ is readily extended to give telescopes

$$
\begin{aligned}
& \operatorname{Tel}(\widetilde{X}) \equiv \operatorname{Tel}(X \stackrel{\text { id }}{\longleftarrow} X \stackrel{\text { id }}{\longleftarrow} \cdot .) \cong X \times R^{+}, \\
& \operatorname{Tel}(\widetilde{Y}) \equiv \operatorname{Tel}(Y \stackrel{\text { id }}{\longleftarrow} Y \stackrel{\text { id }}{\longleftarrow} \cdot .) \cong Y \times R^{+} \text {, } \\
& \operatorname{Tel}(\widetilde{Z}) \equiv \operatorname{Tel}(Y \stackrel{f}{\longleftarrow} X \stackrel{g}{\longleftarrow} Y \stackrel{f}{\longleftarrow} X \stackrel{g}{\longleftarrow} . .) .
\end{aligned}
$$

Also, because the homotopies $H_{n}$ and $K_{n}$ (used to define the maps $F: \operatorname{Tel}(\epsilon(X)$ ) $\rightarrow \operatorname{Tel}(\widetilde{Z})$ and $G^{\prime}: \operatorname{Tel}(\widetilde{Z}) \rightarrow \operatorname{Tel}(\epsilon(Y)$ ) (see formula (2.4) and the following discussion)) are defined on all of $X \times I$ and $Y \times I$ respectively (see conditions (iv) and $(v)$, above) we obtain maps $\widetilde{F}: \operatorname{Tel}(\widetilde{X}) \rightarrow \operatorname{Tel}(\widetilde{Z})$ and $\widetilde{G}^{\prime}: \operatorname{Tel}(\widetilde{Z}) \rightarrow \operatorname{Tel}(\widetilde{Y})$ and a commutative diagram 
(2.8)

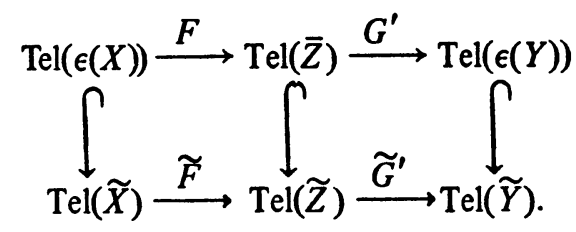

Now let $B$ be a compactum in $Y$. We shall first define an auxiliary map $f^{\prime \prime}: X \rightarrow Y$ depending upon $B$, a homotopy $H: X \times I \rightarrow Y$ with $H_{0}=f^{\prime}$ and $H_{1}=f^{\prime \prime}$, and a compactum $A$ in $X$ such that $H((X \backslash A) \times I) \subset(Y \backslash B)$. Both $H$ and $A$ depend upon $B$. We shall then define a similar homotopy from $f^{\prime \prime}$ to $f$.

To define $f^{\prime \prime}$, choose an integer $n$ so that $Y_{n} \subset(Y \backslash B)$. Let $h_{n}: X \rightarrow R^{+}$ be the map

$$
h_{n}(x)=\min (h(x), n)
$$

where $h$ is the map used to construct a proper section for $\epsilon(X)$. Let $f^{\prime \prime}: X \rightarrow Y$ be the composite $p^{\prime} \widetilde{G}^{\prime} \widetilde{F}\left(x, h_{n}(x)\right)$. (Recall that $p^{\prime}$ is the projection $\operatorname{Tel}(\epsilon(Y)) \rightarrow$ $Y$. See Figure IV.

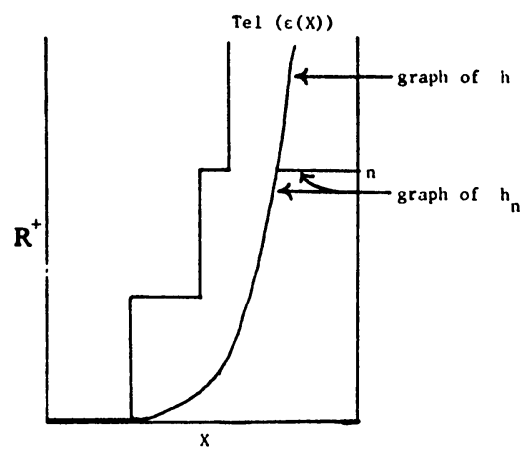

FIGURE IV

The required homotopy is given by

$$
H(x, \tau)=p^{\prime} \circ \widetilde{G}^{\prime} \widetilde{F}\left(x,(1-\tau) \cdot h(x)+\tau \cdot h_{n}(x)\right) .
$$

Then $\left.H\left(X_{n+1} \times I\right) \subset Y_{n}\right)$. If $A$ is the compactum $\operatorname{cl}\left(X \backslash X_{n+1}\right)$, then

$$
H((X \backslash A) \times I) \subset(Y \backslash B),
$$

as required.

It remains to find a compactum $A^{\prime}$ in $X$ and a homotopy $H^{\prime}: X \times I \rightarrow Y$ such that $H_{0}^{\prime}=f^{\prime \prime}, H_{1}^{\prime \prime}=f$, and $H^{\prime}\left(\left(X \backslash A^{\prime}\right) \times I\right) \subset(Y \backslash B)$. To do this, observe that

$$
p^{\prime} \circ \widetilde{G}^{\prime} \widetilde{F}(x, n)=f(x),
$$

so that $H^{\prime}$ may be given by the formula 


$$
H^{\prime}(x, \tau)=p^{\prime} \circ \widetilde{G}^{\prime} \widetilde{F}\left(x,(1-\tau) \cdot h_{n}(x)+\tau \cdot n\right) .
$$

See Figure V. Finally, $H^{\prime-1}(Y \backslash B) \supset H^{\prime-1}\left(Y_{n}\right) \supset X_{n+1} \times I$ so that we may take $A^{\prime}=\operatorname{cl}\left(X \backslash X_{n+1}\right)$.
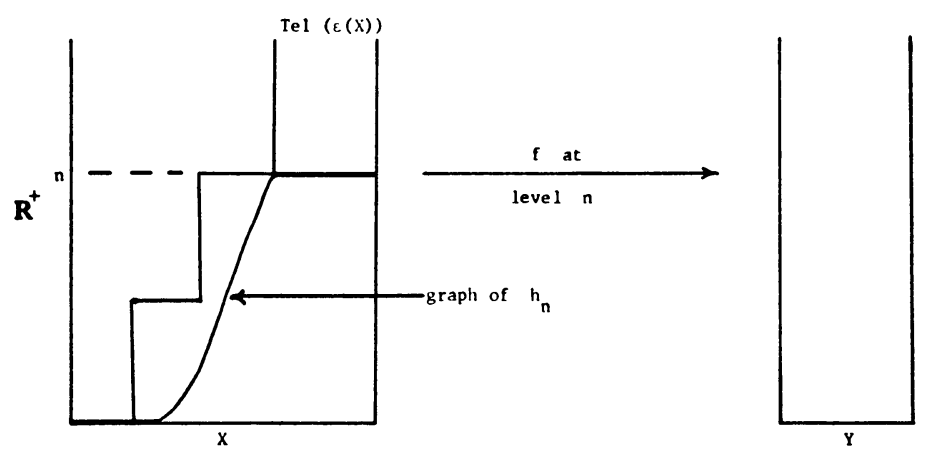

FIGURE V

\section{REFERENCES}

1. E. M. Brown, On the proper homotopy type of simplicial complexes, Lecture Notes in Math., vol. 375, Springer-Verlag, Berlin and New York, 1974.

2. T. A. Chapman, On some applications of infinite-dimensional manifolds to the theory of shape, Fund. Math. 76 (1972), 181-193.

3. D. A. Edwards and H. M. Hastings, Čech and Steenrod homotopy theory, with applications to geometric topology, Lecture Notes in Math., Springer-Verlag, Berlin and New York (to appear).

4. Counterexamples to infinite-dimensional Whitehead theorems in pro-homotopy (to appear).

5. F. T. Farrell, L. R. Taylor and J. B. Wagoner, The Whitehead theorem in the proper category, Compositio Math. 27 (1973), 1-23. MR 48 \#12545.

6. L. C. Siebenmann, Infinite simple homotopy types, Nederl. Akad. Wetensch. Proc. Ser. A 73 = Indag. Math. 32 (1970), 479-495. MR 44 \#4746.

7. T. Chapman, All Hilbert cube manifolds are triangulable (to appear).

8. T. Chapman and L. C. Siebenmann, Finding a boundary for a Hilbert cube manifold (to appear).

9. M. Mather, Counting homotopy types of manifolds, Topology 4 (1965), 93-94. MR 31 \#742; erratum, 35, p. 1577.

10. L. C. Siebenmann, Chapman's classification of shapes-a proof using collapsing, Manuscripta Math. 16 (1975), 373-384.

11. J. E. West, Mapping cylinders of Hilbert cube factors, General Topology and Appl. 1 (1971), 111-125. MR 44 \#5984.

DEPARTMENT OF MATHEMATICAL SCIENCES, SUNY AT BINGHAMTON, BINGHAMTON, NEW YORK 13901

DEPARTMENT OF MATHEMATICS, HOFSTRA UNIVERSITY, HEMPSTEAD, NEW YORK 11550 